p-n junction heterostructure device physics model of a four junction solar cell

Melissa J. Griggs, Brendan M. Kayes, Harry A. Atwater

Melissa J. Griggs, Brendan M. Kayes, Harry A. Atwater, "p-n junction heterostructure device physics model of a four junction solar cell," Proc. SPIE 6339, High and Low Concentration for Solar Electric Applications, 63390D (2 October 2006); doi: 10.1117/12.680793

SPIE. Event: SPIE Optics + Photonics, 2006, San Diego, California, United States 


\title{
p-n Junction Heterostructure Device Physics Model of a Four Junction Solar Cell
}

\author{
Melissa J. Griggs*, Brendan M. Kayes, and Harry A. Atwater \\ California Institute of Technology, Thomas J. Watson, Sr. Laboratories of Applied Physics, MC \\ 128-95, Pasadena, California 91125
}

\begin{abstract}
We present results from a p-n junction device physics model for GaInP/GaAs/GaInAsP/GaInAs four junction solar cells. The model employs subcells whose thicknesses have an upper bound of $5 \mu \mathrm{m}$ and lower bound of $200 \mathrm{~nm}$, which is just above the fully depleted case for the assumed doping of $\mathrm{N}_{\mathrm{A}}=1 \times 10^{18} \mathrm{~cm}^{-3}$ and $\mathrm{N}_{\mathrm{D}}=1 \times 10^{17} \mathrm{~cm}^{-3}$. The physical characteristics of the cell model include: free carrier absorption, temperature and doping effects on carrier mobility, as well as recombination via Shockley-Read-Hall recombination from a single midgap trap level and surface recombination. Upper bounds on cell efficiency set by detailed balance calculations can be approached by letting the parameters approach ideal conditions. However whereas detailed balance calculations always benefit from added subcells, the current matching requirements for series connected $\mathrm{p}-\mathrm{n}$ multi-junctions indicate a minimum necessary performance from an added subcell to yield a net increase in overall device efficiency. For the four junction cell considered here, optimizing the subcell thickness is an important part of optimizing the efficiency. Series resistance limitations for concentrator applications can be systematically explored for a given set of subcells. The current matching limitation imposed by series connection reduces efficiency relative to independently-connected cells. The overall trend indicates an approximately 5\% drop in efficiency for series connected cells relative to identical independently connected cells. The series-connected devices exhibit a high sensitivity to spectral changes and individual subcell performance. If any single subcell within the series-connected device is degraded relative to its optimal design, the entire device is severely hindered. This model allows complex heterostructure solar cell structures to be evaluated by providing device physics-based predictions of performance limitations.
\end{abstract}

Keywords: Multi-junction; solar cells; modeling; device physics

\section{INTRODUCTION}

Multi-junction compound semiconductor solar cells have consistently demonstrated performance as the highest efficiency cells due to their ability to absorb a greater portion of the solar spectrum more effectively (see Fig. 1). High efficiency triple junction solar cells have recently been produced with efficiencies of approximately $39 \%{ }^{1}$. To achieve the highest efficiencies, the optoelectronic properties of the materials used in multi-junction solar cells must be optimized to efficiently absorb and convert as much of the solar flux as possible. One of the primary difficulties with these devices is evaluating new design approaches. Device physics based modeling has been performed for GaInP/GaAs multijunction devices ${ }^{2}$, but currently most modeling of complex (>2 junctions) III-V multi-junction solar cells is based on detailed balance calculations ${ }^{3-5}$. Detailed balance models provide an ultimate upper bound for the structure modeled from the thermodynamic limit. Much as a Carnot engine is the upper bound for heat engines, these calculations cannot inherently provide any information about the realistic performance of any given cell structure. For example, according to detailed balance calculations, the addition of subcells always improves the performance of the cell. However, poor quality subcells can actually hinder the overall heterostructure device.

Models of single junction silicon solar cells have been developed with good models for the sources of non-ideality in those cells. The most commonly used of these are AMPS and PC1D $\mathrm{D}^{6,7}$. These models have been extended over time to include more sophisticated understandings of the physics involved in silicon solar cells. Unfortunately, the main complication of multi-junction solar cell modeling is imposing current matching between the cells to satisfy the series

*griggs@caltech.edu

High and Low Concentration for Solar Electric Applications, edited by Martha Symko-Davies Proc. of SPIE Vol. 6339, 63390D, (2006) · 0277-786X/06/\$15 - doi: 10.1117/12.680793 
connection requirements. In addition, the wealth of experimental data that is available for silicon is not available for many of the III-V materials of interest. For these reasons, there has been great interest in realistic models of III-V multijunction models. A few such models have been created, the commercial software from Silvaco, ATLAS ${ }^{8}$, has been shown to be effective in modeling two-dimensional photogeneration and providing similar IV characteristics to experimental solar cells. In addition, the model Syracuse ${ }^{9}$ has been developed to effectively model the performance of any III-V multi-junction solar cell anywhere in the world. The model includes an extensive library of spectral irradiance data experimental and calculated. However, neither of these models allows for the optimization of the device structure. With current matching limitations, the thicknesses of each of the subcells are extremely important to ensure the best performing cell possible. A group in Kuwait has developed a model ${ }^{10}$ that allows for the optimization of various parameters of the cell, although they have to make many approximations to model the effects that are seen experimentally.

In this work, we have developed a model that includes as many numerical models for the behaviors of III-V compound semiconductors as a function of composition, temperature, and doping concentrations. In this way, we hope to avoid unnecessary assumptions and allow ourselves the flexibility to model any III-V compound semiconductor. In addition, we have incorporated the flexibility to evaluate electrical connections other than the fully series connected stack.

As we are developing a four junction cell in our lab using wafer bonding and layer transfer, we have been modeling that cell as a starting point, though the code can be applied to any III-V materials. Therefore, all the data in this paper will be of the GaInP/GaAs/GaInAsP/GaInAs four-junction cell. The top two cells are lattice-matched to GaAs and the bottom two cells are lattice-matched to InP.

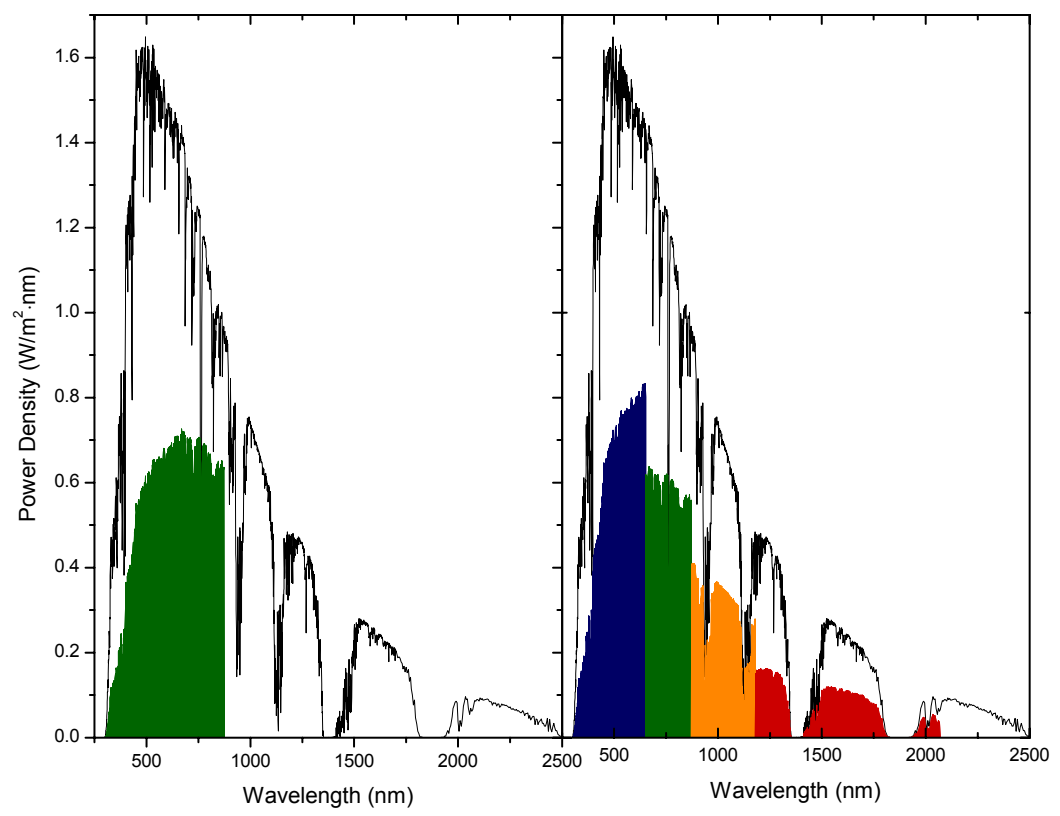

Figure 1. Power density as a function of wavelength, the shaded regions denote the power density of a single junction GaAs cell (left) and that of a multi-junction $\mathrm{GaInP} / \mathrm{GaAs} / \mathrm{GaInAsP} / \mathrm{GaInAs}$ cell (right).

\section{THE MODEL}

The overall current density is given by combination of the diffusion and drift equations ${ }^{11}$. When paired with the continuity conditions and Gauss' Law, and assuming Shockley-Read-Hall recombination from a single trap at midgap as well as exponential light absorption, the current density in each subcell can be solved to give the solution shown in equations 1-10. The p-n junction is assumed to be abrupt, which is common in CVD grown cells, and the depletion 
approximation was assumed to be valid. The emitter layer is assumed to be n-type, while the base is p-type. Each cell is divided into four regions, the quasineutral region of the emitter (thickness $=\mathrm{x}_{1}$ ), the depletion region of the emitter (thickness $=\mathrm{x}_{2}$ ), the depletion region of the base (thickness $=\mathrm{x}_{3}$ ), and the quasi-neutral region of the base (thickness $=$ $\left.\mathrm{x}_{4}\right)$.

$$
J=\left(J_{0}^{p}+J_{0}^{n}\right)\left(e^{q V / k T}-1\right)-J_{l}^{p}-J_{l}^{n}-J_{d e p}^{g}(V)+J_{r}^{d e p}(V)
$$

Where

$$
\begin{gathered}
J_{0}^{n}=-\left(\frac{q D_{p}}{L_{p}}\right) p_{0}\left(\frac{\left(S_{p} L_{p} / D_{p}\right) \cosh \left(x_{1} / L_{p}\right)+\sinh \left(x_{1} / L_{p}\right)}{\left(S_{p} L_{p} / D_{p}\right) \sinh \left(x_{1} / L_{p}\right)+\cosh \left(x_{1} / L_{p}\right)}\right) \\
J_{0}^{p}=-\left(\frac{q D_{n}}{L_{n}}\right) n_{0}\left(\frac{\left(S_{n} L_{n} / D_{n}\right) \cosh \left(x_{4} / L_{n}\right)+\sinh \left(x_{4} / L_{n}\right)}{\left(S_{n} L_{n} / D_{n}\right) \sinh \left(x_{4} / L_{n}\right)+\cosh \left(x_{4} / L_{n}\right)}\right) \\
J_{l}^{n}=-\left(\frac{q \Gamma_{0}}{1-\alpha^{-2} L_{p}^{-2}}\right)\left\{\frac{\left(S_{p} / \alpha D_{p}\right)+1-\left[\left(S_{p} / \alpha D_{p}\right) \cosh \left(x_{1} / L_{p}\right)+\left(1 / \alpha D_{p}\right) \sinh \left(x_{1} / L_{p}\right)\right] e^{-\alpha x_{1}}}{\left(S_{p} L_{p} / D_{p}\right) \sinh \left(x_{1} / L_{p}\right)+\cosh \left(x_{1} / L_{p}\right)}-e^{-\alpha x_{1}}\right\} \\
J_{l}^{p}=-\left(\frac{q \Gamma_{0} e^{-\alpha(x 1+x 2+x 3)}}{1-\alpha^{-2} L_{n}^{-2}}\right)\left\{1-\frac{1}{\alpha L_{n}}\left[\frac{\left(S_{n} L_{n} / D_{n}\right)\left(\cosh \left(x_{4} / L_{n}\right)-e^{-\alpha x_{4}}\right)+\sinh \left(x_{4} / L_{n}\right)+\alpha L_{n} e^{-\alpha x_{4}}}{\left(S_{n} L_{n} / D_{n}\right) \sinh \left(x_{4} / L_{n}\right)+\cosh \left(x_{4} / L_{n}\right)}\right]\right\} \\
J_{r}^{\text {dep }}(V)=-\frac{q n_{i}\left(x_{2}+x_{3}\right)}{\sqrt{\tau_{n 0} \tau_{p 0}}}\left[\frac{2 \sinh (q V / 2 k T)}{q\left(V_{b i}-V\right) / k T}\right] \frac{\pi}{2}
\end{gathered}
$$

Where

$$
\begin{aligned}
& x_{2}(V)=\left(\frac{2 N_{A} \varepsilon\left(V_{b i}-V\right)}{q N_{D}\left(N_{D}+N_{A}\right)}\right)^{1 / 2} \\
& x_{3}(V)=\left(\frac{2 N_{D} \varepsilon\left(V_{b i}-V\right)}{q N_{A}\left(N_{D}+N_{A}\right)}\right)^{1 / 2} \\
& V_{b i}=\frac{k T}{q} \log \left(\frac{N_{A} N_{D}}{n_{i}^{2}}\right)+\frac{\Delta E_{c}}{q}
\end{aligned}
$$

Where $q$ is electronic charge, $D_{n / p}$ is the diffusivity of carriers, $L_{n / p}$ is the diffusion length of carriers, $S_{n / p}$ is the surface recombination velocity, $n_{0} / p_{0}$ are the equilibrium concentrations of minority carriers, $\Gamma_{0}$ is the solar flux hitting the top of the subcell, $\alpha$ is the absorption coefficient of the material, $\varepsilon$ is the static dielectric constant of the material, $n_{i}$ is the 
intrinsic carrier concentration, $\tau_{n 0 / p 0}$ is the extreme minimum lifetime for carriers when all the recombination centers are filled with carriers of the opposite sign, $\Delta E_{c}$ is the conduction-band offset, and $k$ is the Boltzmann constant.

The current-bias curves are calculated for each individual cell using the equations above and an incident solar flux that is filtered by the absorption of the cells above. Then current matching is enforced ${ }^{12}$ (eqn. 11).

$$
V(J)=\sum_{i=1}^{m} V_{i}(J)
$$

As one of the main questions in concentrator multi-junction solar cells is the effect of temperature and doping concentration on performance. Currently, we include a Drude model for free-carrier absorption ${ }^{13}$ (see eqn. 12)

$$
\alpha_{f c a}=\frac{q^{2} \lambda^{2} n_{c}}{4 \pi^{2} c^{3} \varepsilon_{0} n_{r} m_{c} \tau_{c}}
$$

Where

$$
\tau_{c}=\frac{m_{c} \mu_{c}}{q}
$$

Where $\mathrm{q}$ is electronic charge, $\lambda$ is the photon wavelength, $\mathrm{n}_{\mathrm{c}}$ is the carrier concentration, $n_{r}$ is the refractive index, $m_{c}$ is the effective mass of the carriers, $\varepsilon_{0}$ is the permittivity of free space, and $\mu_{c}$ is the carrier mobility. In the future, we plan in include a more sophisticated model for free carrier absorption that includes inter and intra-valley absorptions processes $^{13}$. For the time being, the Drude model provides an over-estimate of free carrier absorption. The material constants are shown in table 1.

Table 1. Material constants used in the model.

\begin{tabular}{lccccccc}
\hline \multicolumn{1}{c}{ Material } & $\mathrm{n}_{\mathrm{r}}$ & $\begin{array}{c}\mathrm{n}_{\mathrm{i}} \\
\left(\mathrm{cm}^{-3}\right)\end{array}$ & $\mathrm{m}_{\mathrm{n}}$ & $\mathrm{m}_{\mathrm{p}}$ & $\begin{array}{c}\mathrm{S}_{\mathrm{n}} \\
(\mathrm{cm} / \mathrm{s})\end{array}$ & $\begin{array}{c}\mathrm{S}_{\mathrm{p}} \\
(\mathrm{cm} / \mathrm{s})\end{array}$ & $\varepsilon$ \\
\hline $\mathrm{Ga}_{0.51} \mathrm{In}_{0.49} \mathrm{P}$ & 11.8 & $1.99 \mathrm{E}+02$ & 0.067 & 0.7 & 4000 & 1.5 & 11.8 \\
$\mathrm{GaAs}$ & 12.9 & $2.10 \mathrm{E}+06$ & 0.088 & 0.51 & 1000 & 1000 & 12.9 \\
$\mathrm{Ga}_{0.19} \mathrm{In}_{0.81} \mathrm{As}_{0.37} \mathrm{P}_{0.63}$ & 13.4 & $2.75 \mathrm{E}+09$ & 0.041 & 0.48 & 1000 & 1000 & 13.4 \\
$\mathrm{Ga}_{0.47} \mathrm{In}_{0.53} \mathrm{As}$ & 13.9 & $1.63 \mathrm{E}+12$ & 0.059 & 0.45 & 1000 & 1000 & 13.9 \\
\hline
\end{tabular}

In addition, we have accounted for the temperature and doping concentration effects on carrier mobility using the empirical model developed by Sotoodeh et al. ${ }^{14}$ (see eqn. 14).

$$
\mu_{L F}(N, T)=\mu_{\min }+\frac{\mu_{\max }(300 K)(300 K / T)^{\theta_{1}}-\mu_{\text {min }}}{1+\left(\frac{N}{N_{r e f}(300 K)(T / 300 K)^{\theta_{2}}}\right)}
$$

Where all the constants are fit parameters and $N$ is the carrier concentration and $T$ is the temperature. The parameters used in this model are shown in Table 2. With a good fit to those materials that are well understood, this provides a reasonable estimate for all four materials used in this study.

One of the largest challenges in a multijunction device model involves the limitations that current matching imposes. When a multi-junction solar cell is made up of series connected subcells, the subcell thicknesses have a large impact on the device performance (see Fig. 2). Changing the thicknesses of the cell adjusts which cell is the current-limiting cell. In this way, a thorough understanding of the effects of diffusion length on the overall cell performance cannot be obtained without optimizing the subcell thicknesses for each set of conditions. Unfortunately, this is a difficult 
multivariate optimization routine with many local optima in the surface. All the data presented within this paper were calculated to be as close to the optimum value as possible, but are probably only near-optimum. However, the optimization routine is run with many different starting points to ensure that the entire surface is explored. All trends are definitely correct, and the maximum efficiency values are very close (within .1\%) to the true optimums.

Table 2. Fit parameters for each material used in the mobility model.

\begin{tabular}{lccccccc}
\hline \multicolumn{1}{c}{ Material } & $\begin{array}{c}\text { Electron } \\
\text { or hole }\end{array}$ & $\begin{array}{c}\mu_{\max }(300 \mathrm{~K}) \\
\left(\mathrm{cm}^{2} / \mathrm{Vs}\right)\end{array}$ & $\begin{array}{c}\mu_{\min } \\
\left(\mathrm{cm}^{2} / \mathrm{V} \mathrm{s}\right)\end{array}$ & $\begin{array}{c}\mathrm{N}_{\text {ref }}(300 \mathrm{~K}) \\
\left(\mathrm{cm}^{-3}\right)\end{array}$ & $\lambda$ & $\theta_{1}$ & $\theta_{2}$ \\
\hline $\mathrm{Ga}_{0.51} \mathrm{In}_{0.49} \mathrm{P}$ & electron & 4300 & 400 & $2.0 \mathrm{E}+16$ & 0.70 & 1.66 & 1.95 \\
$\mathrm{GaAs}$ & hole & 150 & 15 & $1.50 \mathrm{E}+17$ & 0.80 & 2.0 & 1.47 \\
& $\begin{array}{c}\text { electron } \\
\mathrm{G}\end{array}$ & 9400 & 500 & $6.0 \mathrm{E}+16$ & 0.394 & 2.1 & 3.0 \\
$\mathrm{Ga}_{0.19} \mathrm{In}_{0.81} \mathrm{As}_{0.37} \mathrm{P}_{0.63}$ & hole & 492 & 20 & $1.48 \mathrm{E}+17$ & 0.38 & 2.2 & 3.0 \\
& electron & 3771 & 99 & $9.76 \mathrm{E}+16$ & 0.517 & 1.39 & 3.01 \\
$\mathrm{Ga}_{0.47} \mathrm{In}_{0.53} \mathrm{As}$ & hole & 104 & 13 & $2.36 \mathrm{E}+17$ & 0.606 & 1.58 & 2.64 \\
& electron & 14000 & 300 & $1.3 \mathrm{E}+17$ & 0.48 & 1.59 & 3.68 \\
& hole & 320 & 10 & $4.9 \mathrm{E}+17$ & 0.403 & 1.59 & 3.0 \\
\hline
\end{tabular}

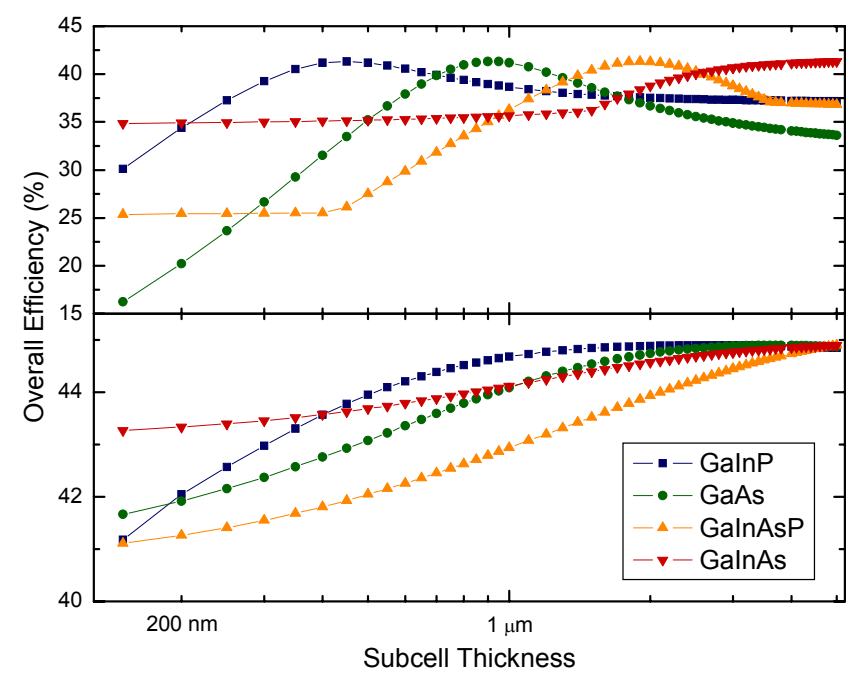

Figure 2. Overall cell efficiency as a function of subcell thickness. The subcell thicknesses were all set to the nearoptimum values for the series connected cell in the top graph and the independently connected cell in the bottom graph; then each subcell thickness was varied individually demonstrating the near-optimum values that are obtained in the series connected case.

\section{RESULTS}

Before testing our model full four junction solar cell heterostructures, we performed simpler preliminary calculations to verify that the physical parameters and models made sense. To do this, we varied the diffusion lengths in one subcell at a time and looked at the effect on the individual subcell short circuit current and open circuit voltage (Fig. 3). As expected, the open circuit voltage drops off before the short circuit current with a reduction in the diffusion length. 


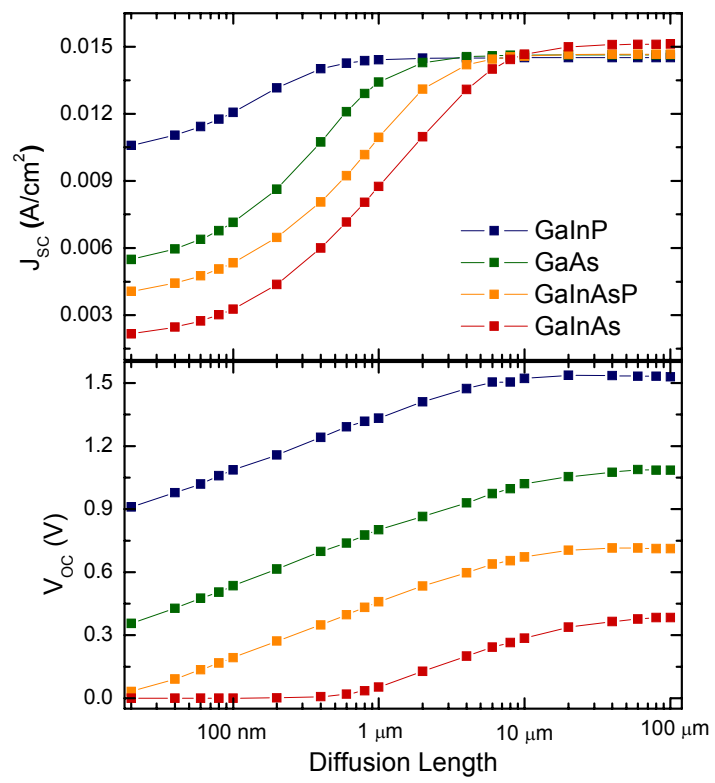

Figure 3. Short circuit current $\left(\mathrm{J}_{\mathrm{SC}}\right)$ and open circuit voltage $\left(\mathrm{V}_{\mathrm{OC}}\right)$ of the individual subcells as a function of the subcell diffusion length.
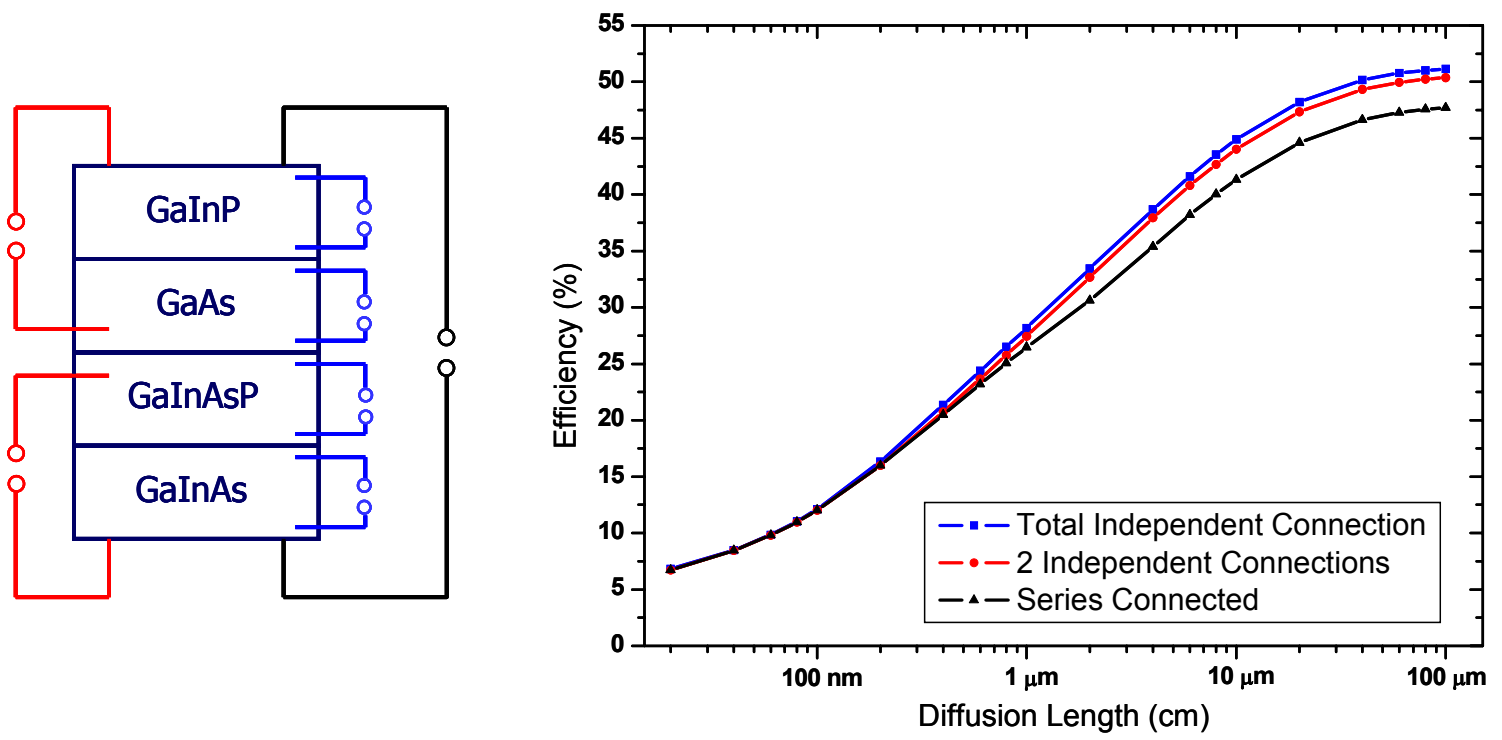

Figure 4. Overall cell efficiency as a function of the diffusion length in all four subcells for 3 different electrical connections.

We explored the effect of the electrical connection configuration (series or independent) on cell performance. It is well known that series connection can limit cell performance, but we wanted to understand the magnitude of the effect, and whether an independent electrical configuration would provide sufficient gain to justify the added cost and complexity. Therefore, we considered the three different electrical connections shown in Figure 4: the fully series connected device, a device with 2 independent connections separating the top two subcells from the bottom two, and the fully independently connected device. The first test we ran was to co-vary the diffusion lengths in all four subcells together 


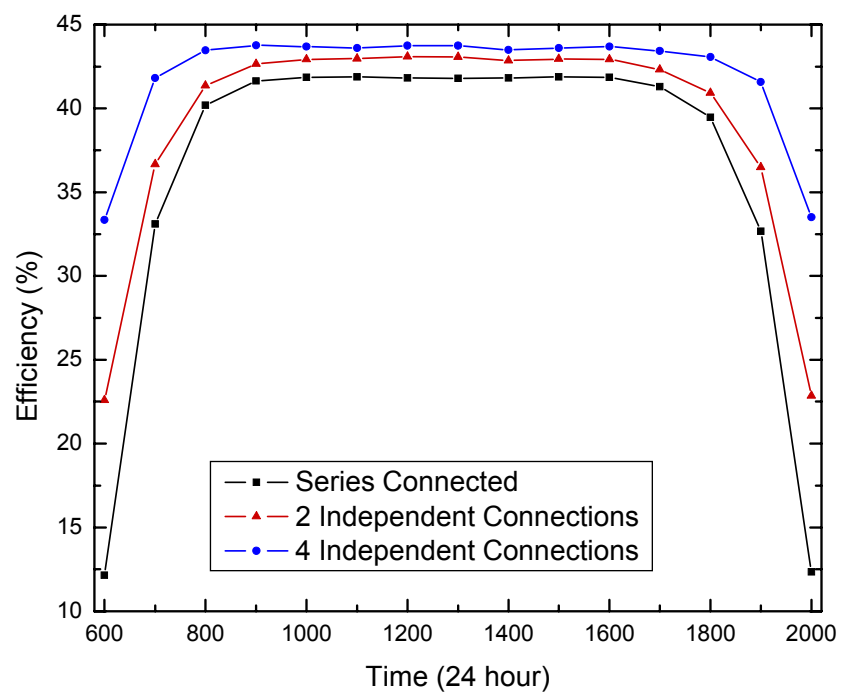

Figure 5. Overall cell efficiency as a function of time, using the reference fluxes for a Hot Sunny Day.

and look at the overall device efficiency (see Fig. 4). Interestingly, the 2 independent connections configuration gives $75 \%$ of the gain achieved from total independent connections, and is more feasible.

In addition, we can explore the electrical connection effect further by using the reference Hot Sunny Day ${ }^{15}$ spectral data to simulate the overall power output change in a full day/night cycle. By optimizing the overall cell of each type for the peak flux of the day, then calculating the performance of these devices over the course of the day we can elucidate the true differences in these solar cells. As can be seen in Fig. 5, the efficiency roll off in the early and late hours of the day is much less significant in the four independent connections cell. In terms of power density produced, the four independent connections cell provides a $\sim 9 \%$ gain, and the 2 independent connections cell produces $\sim 60 \%$ of that gain.

\section{CONCLUSIONS}

We have developed a model for simulating the performance of complex ( $>2$ junction) III-V multi-junction solar cells. The model allows us to explore the complex multivariate space that governs device performance to better understand which parts of the device will benefit most from improvement. We have explored the creation of a 2 independent connection device that indicates a benefit relative to a series connected structure.

\section{ACKNOWLEDGEMENTS}

This work was supported by the High Performance Photovoltaic Program of the National Renewable Energy Laboratory under subcontract XAT-4-33624-10. One of us (MJG) acknowledges the National Science Foundation for a Graduate Research Fellowship as well as J. Dionne and M. Kelzenberg for many discussions on implementation.

\section{REFERENCES}

1. King, R., et al., Pathways to 40\%-efficient concentrator photovoltaics. Proceedings of the 20th European Photovoltaic Solar Energy Conference, 2005. 
2. McMahon, W.E., et al., An On-Sun Ccomparison of GaInP2/GaAs Tandem Cells with Top Cell Thickness Varied. Proceedings of the 31st IEEE Photovoltaic Specialists Conference, 2005: p. 715-718.

3. Shockley, W. and H.J. Quiesser, Detailed Balance limit of efficiency of p- $n$ junction solar cells. Journal of applied physics, 1961. 32(3): p. 510-519.

4. Henry, C.H., Limiting Efficiencies of ideal single and multiple energy gap terrestrial solar cells. Journal of Applied Physics, 1980. 51(8): p. 4494-4500.

5. Araujo, G. and A. Marti, Absolute limiting efficiencies for photovoltaic energy conversion. Solar Energy Materials and Solar Cells, 1994. 33: p. 213-240.

6. Arch, J.K., et al., Computer-Analysis of the Role of P-Layer Quality, Thickness, Transport Mechanisms, and Contact Barrier Height in the Performance of Hydrogenated Amorphous-Silicon P-I-N Solar-Cells. Journal of Applied Physics, 1991. 69(10): p. 7057-7066.

7. Clugston, D.A. and P.A. Basore, PC1D Version 5: 32-bit Solar Cell Modeling on Personal Computers. Proceedings of the 26th IEEE Photovoltaic Specialists Conference, 1997: p. 207-210.

8. Michael, S., A.D. Bates, and M.S. Green, Silvaco ATLAS as a Solar Cell Modeling Tool. Proceedings of the 31st IEEE Photovoltaic Specialists Conference, 2005: p. 719-721.

9. Ekins-Daukes, N.J., et al., Syracuse - A Multi-junction Conecntrator System Computer Model. Proceedings of the 31st IEEE Photovoltaic Specialists Conference, 2005: p. 651-654.

10. Ghannam, M.Y., et al., Optimization of the triple junction In0.5Ga0.5P/GaAs/Ge monolithic tandem cell aimed for terrestrial applications using an experimentally verified analytical model. Kuwait Journal of Science \& Engineering, 2004. 31(2): p. 203-234.

11. Fahrenbruch, A.L. and R.H. Bube, Fundamentals of Solar Cells. 1983, London: Academic Press, Inc.

12. Luque, A. and S. Hegedus, eds. Handbook of Photovoltaic Science and Engineering. 2003, John Wiley \& Sons, Ltd.: Chichester, West Sussex, England.

13. Tsai, C.Y., et al., Theoretical model for intravalley and intervalley free-carrier absorption in semiconductor lasers: Beyond the classical Drude model. Ieee Journal of Quantum Electronics, 1998. 34(3): p. $552-559$.

14. Sotoodeh, M., A.H. Khalid, and A.A. Rezazadeh, Empirical low-field mobility model for III-V compounds applicable in device simulation codes. Journal of Applied Physics, 2000. 87(6): p. 2890-2900.

15. Marion, B., et al., Validation of a Photovoltaic Module Energy Ratings Procedure at NREL. NREL Technical Report 520-26909, 1999. 